\title{
Clinical radiation oncology trials in Germany
}

\author{
Time for clarification and professionalization
}

\author{
Rainer Fietkau · Frederik Wenz
}

Published online: 2 November 2015

(C) Springer-Verlag Berlin Heidelberg 2015

Radiotherapy is a mainstay of modern oncology. Improvements in radiotherapy have in the past and will in the future contribute substantially to the progress made in cancer treatment. Clinical trials are the basis for further advancing radiotherapeutic efficacy to improve locoregional tumor control and/or to better protect normal tissues, i.e., to improve the therapeutic ratio [1]. It is therefore of central importance for current and future cancer patients to promote clinical trials in radiation oncology. This requires innovative and patient-oriented ideas brought forward by dedicated clinical scientists or study groups, professional trial infrastructures and appropriate funding, but also a supportive medicolegal framework. In Germany this latter point differs in several important aspects from the requirements for clinical trials in radiation oncology in other states of the European Union and worldwide.

Application of ionizing radiation for the purpose of medical research in Germany needs to be approved not only by the institutional review boards of participating center but also by the national authority for radiation protection (Bundesamt für Strahlenschutz, BfS). For studies in the field of radiation oncology, particularly the differentiation between use of radiation for "medical care (Heilkunde)" versus "medical research" frequently leads to contradic-

Prof. Dr. R. Fietkau ( $₫)$

Strahlenklinik, Universitätsklinikum Erlangen,

Erlangen, Germany

e-mail: rainer.fietkau@uk-erlangen.de

Prof. Dr. F. Wenz

Klinik für Strahlentherapie und Radioonkologie,

Universitätsklinikum Mannheim,

Mannheim, Germany

e-mail: frederik.wenz@medma.uni-heidelberg.de tions and confusion. Clinical trials have been implemented slower than expected, some international clinical trials have avoided participation of German centers due to the required elaborate approval procedure, and in other cases accrual of international trials was far advanced or even completed before German centers were initiated. Such complicated processes bear great risk that German cancer patients miss the chance to participate in innovative trials, that clinical radiation oncology research in Germany falls behind compared to research in other countries or in other fields of oncology, and that innovation in Germany may be implemented without proper assessment outside clinical trials.

It is therefore of significant value for future clinical research in radiation oncology that Simon and Habeck et al. summarize in a comprehensive, easy to understand manuscript in this issue of Strahlentherapie und Onkologie the medicolegal framework for trials using radiation in Germany [2]. The paper aims to provide principle investigators, individuals, and institutions involved in the planning process of clinical trials a fundamental understanding of the key terms "medical care" and "medical research." It clearly indicates in which situation trials need to be approved by the BfS and which trials can be performed under the usual professional laws after review by the institutional ethics committee without involvement of the BfS. Particularly important is that the paper by Simon and Habeck et al. is not only authored by experienced principle investigators, trial coordinators, and medical law experts but also by experts from the BfS. It has been approved as an official statement by the German Society for Radiation Oncology (DEGRO) and will serve as a valuable practice guideline for principle investigators, institutions, and ethics committees. 
The most important results and conclusions of the review are the following:

- The key question for differentiating between "medical care (Heilkunde)" which does not need to be approved by the BfS and "medical research" which needs to be approved by the BfS is whether the specific application of radiation (beam quality, dose, schedule, target volume, etc.) is a clinically established and recognized procedure ("klinisch anerkanntes Strahlentherapieverfahren") or not.

- This question needs to answered by the qualified physician(s) ("fachkundiger Arzt" according to German radiation protection law) in charge of treating patients within the trial, under consideration of the best available evidence from clinical studies, as well as of guidelines, textbooks, or consensus papers by the relevant scientific societies. Indication, total dose, fractionation, and target volume definition are among the important parameters for the assessment.

- For the decision whether a trial needs to be approved by the BfS, it is not relevant whether a treatment is already available at other institutions or whether it is accepted for re-imbursement.

- The decision-making by the qualified physician(s) ("fachkundiger Arzt") may be supported on request by an opinion given by the DEGRO Expert Committee for clinical trials.

- Correct assessment at an early stage whether a trial needs to be approved by the BfS or not may reduce unnecessary costs and reduce the time needed for the approval procedures.

Simon and Habeck et al. give examples of trials in an appendix to the paper which necessitate approval by the BfS and of those which do not. For example the national German PREFERE trial which randomly evaluates in early prostate cancer the value of prostatectomy vs. external beam radiotherapy vs. permanent seed implantation vs. active surveillance does not need approval, as all four treatment arms are established treatments for this disease in Germany and randomization per se does not require approval. In contrast, a radiation dose-escalation trial beyond the currently applied level in the same disease would need approval. A trial adding a new chemotherapeutic drug to a standard radiotherapy schedule does not necessarily need approval by the BfS, whereas a novel hypofractionated treatment schedule would need approval.
The paper by Simon and Habeck et al. was already instrumental for the recent update on the statement on proton therapy issued by DEGRO ([3], Strahlentherapie and Onkologie 191 (Vol 8), 2015) in conformance with the hitherto existing position of the BfS. Proton therapy, as a therapeutic option, established at an increasing number of centers worldwide, may be applied within trials without approval by the BfS under certain requirements, including approved indication for radiotherapy, target volume, total dose, dose per fraction, and overall treatment time correspond to the state-of-the-art in photon therapy. The quality of the proton treatment plan must be at least as good as a state-of-the-art conformal photon-based treatment plan and follow-up by the radiation oncologist needs to be assured.

An important aim for promoting clinical research in radiation oncology is to further professionalize planning and implementation of clinical trials in this field. For this, education of current and future PIs, dedicated trial centers for radiation oncology and easy access to medicolegal advice, e.g., within the framework of the German Cancer Society (DKG/ARO) or German Cancer Consortium (DKTK), needs to be strengthened. The paper of Simon and Habeck et $a l$. is an important contribution in this direction. In contrast to many medical trials testing new drugs, this infrastructure and expertise needs to be mainly established by academic institutions as industry rarely supports radiotherapy trials. Close cooperation between such professional centers and large trial networks such as the Radiation Oncology Group (ARO) of the German Cancer Society is of special interest to offer such resources for all clinical trials in need.

Rainer Fietkau, Erlangen, and Frederik Wenz, Mannheim

\section{References}

1. Dörr W et al (2014) Application of organ tolerance dose-constraints in clinical studies in radiation oncology. Strahlenther Onkol 190:621-624, 626-627

2. Simon M, Habeck $M$ et al (2015) Genehmigungsverfahren klinischer Studien im Bereich der Radioonkologie. Strahlenther Onkol. doi:10.1007/s00066-015-0914-3

3. DEGRO (2015) Stellungnahme zur Strahlentherapie mit Protonen in Deutschland. http://www.degro.org/dav/html/aktuelles/201506 StellungnahmeProtonen.pdf. Accessed 25 Sept 2015 